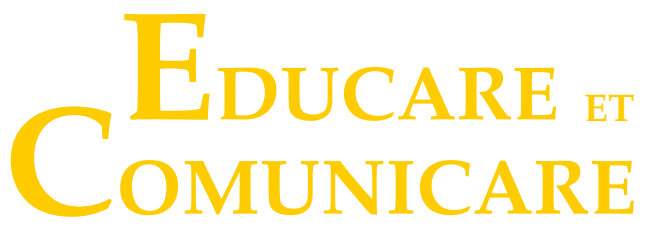

REVISTA CIENTÍFICA DE LA FACULTAD DE HUMANIDADES

https://DOI 10.35383/educare.v9i2.673

e-ISSN: 2312-9018

Universidad Católica Santo Toribio de Mogrovejo

\title{
Formación de competencias ciudadanas para responder a un mundo global
}

\section{Training citizen competences for a global world}

\author{
Segundo Juan Sánchez Tarrillo *1a \\ jsanchez832@gmail.com \\ https://orcid.org/0000-0001-7810-4625
}

\section{Silvia Josefina Aguinaga Vásquez ${ }^{1 \mathrm{a}}$ \\ silviaguinaga25@gmail.com}

https://orcid.org/0000-0002-6763-760X

\author{
*Autor corresponsal \\ 1 Colegio de Profesores del Perú \\ a Magister en Educación
}

\section{Fechas importantes}

Recibido: 05/12/2021

Aceptado:09/12/2021

Publicado online: $31 / 12 / 2021$

\section{Resumen}

La sociedad actual atraviesa una serie de transformaciones que incluyen incertidumbres, cambios acelerados que, en cierto modo, también generaron respuestas y consecuencias en una sociedad dividida, fragmentada, intolerante y poco empática; por eso, más que nunca, se necesita ciudadanos responsables que permitan mejorar la sociedad. Este artículo es el informe de una investigación cuyo propósito fue desarrollar un análisis sistemático de la formación y desarrollo de competencias ciudadanas para responder a un mundo globalizado. La investigación fue realizada por medio de una revisión sistemática con carácter meta analítico de diversas publicaciones, para ello se utilizó proveedores de información y múltiples bases de datos científicas indexadas en: ProQuest, Ebsco, SciELO, Scopus, Concytec, Dialnet, entre otras. De la misma manera, utilizando términos y operadores booleanos, se realizó una Metabúsqueda en el Google Scholar de informes y repositorios publicados por organismos e instituciones internacionales y nacionales como: UNESCO, CEPAL, UNICEF, INEI, CADE-IPAE. Los artículos e informes analizados describen problemas globales, ofrecen diversas estrategias didácticas como pedagógicas para mejorar la convivencia y desarrollar las competencias cívicas de los ciudadanos. Asimismo, se consideraron los criterios de inclusión de fuentes confiables de los últimos cinco años, así como la exclusión de algunos artículos por no estar sujeta a validez y confiabilidad. Finalmente, se concluye que la mayoría de investigadores han publicado estudios con el fin de desarrollar una cultura de paz. Ante esto, los informes que se destacan son cuantitativos, cualitativos y mixtos, muchos de los cuales buscan fortalecer las competencias ciudadanas en diferentes niveles: básico y superior, destacando a Colombia como el país con mayor impacto en las publicaciones de formación de competencias ciudadanas.

Palabras clave: formación básica, competencias para la vida, habilidad ciudadana, competencias ciudadanas, ciudadanía global y educación global. 


\begin{abstract}
Today's society is going through a series of transformations that include uncertainties, accelerated changes that, in a certain way, also generated responses and consequences in a divided, fragmented, intolerant and not very empathetic society; for this reason, more than ever, responsible citizens are needed to improve society. This article is the report of a research whose purpose was to develop a systematic analysis of the formation and development of civic competencies to respond to a globalized world. The research was carried out by means of a systematic review with a meta-analytical nature of various publications, for which information providers and multiple scientific databases indexed in: ProQuest, Ebsco, SciELO, Scopus, Concytec, Dialnet, among others, were used. In the same way, using Boolean terms and operators, a meta-search was carried out in Google Scholar of reports and repositories published by international and national organizations and institutions such as: UNESCO, CEPAL, UNICEF, INEI, CADE-IPAE. The articles and reports analyzed describe global problems, offer various didactic and pedagogical strategies to improve coexistence and develop citizens' civic skills. Likewise, the inclusion criteria of reliable sources from the last five years were considered, as well as the exclusion of some articles for not being subject to validity and reliability. Finally, it is concluded that most researchers have published studies in order to develop a culture of peace. Given this, the reports that stand out are quantitative, qualitative and mixed, many of which seek to strengthen citizenship competencies at different levels: basic and superior, highlighting Colombia as the country with the greatest impact in publications on the formation of citizenship competencies.
\end{abstract}

Keywords: basic training, life skills, citizenship skills, global citizenship and global education.

\title{
Introducción
}

El siglo XXI, es una época cargada de incertidumbre e imprevisibilidad del futuro, de grandes transformaciones, cambios complejos, así como repentinos que generan desequilibrios económicos, sociales, sanitarios, ambientales y políticos CEPAL (2020). Sin duda el 2020 será recordado en la historia como el año que generó una crisis global, nublada por la sombra de una pandemia llamada CORONA COVID-19 (HuancaArohuanca et al., 2020) que va acompañada de fuerzas transformadoras que repercute en la vida del hombre. A nivel global, las naciones están tratando de asegurar que sus programas educativos respondan a los retos que esta exige, aguarda un modelo que entienda a la humanidad y la complejidad del ser humano como un todo; por ello, se requiere enfrentar esta nueva época, teniendo en cuenta un nuevo paradigma en el que no solo se desarrolle las competencias cognitivas, sino también las de naturaleza no cognitiva, con el fin de atender los problemas de ciudadanía y llegar al éxito o como lo refiere CADE Educación (2019) formar profesionales altamente empleables que responda a los retos de la sociedad actual.

Sin embargo, se observa que algunos estudiantes de nivel básico y superior presentan carencias de competencias ciudadanas (Cabrera-Jiménez, 2020), falta de apropiación de competencias cívicas que la mayoría de estudiantes manifiestan en su práctica cotidiana (Alvarez et al., 2017). Por ejemplo: intolerancia en las relaciones interpersonales, presencia de estereotipos marcados que dificultan el reconocimiento del otro (Zambrano, 2018), resistencia permanente a aceptar posturas diversas frente a objetivos comunes (Alvarez et al., 2017), escasa apertura y compromiso de los estudiantes a participar en temas políticos fundamentales (Manuel, 2017), casos de corrupción, desconocimiento de derechos y deberes legales, poco interés en participar en la vida política, percepción de que las leyes se pueden cambiar fácilmente, autoritarismo de algunas naciones, desigualdad social (León, 2020). Por tanto, es fundamental reconocer que la construcción de ciudadanía en todo ser humano no es innata, se educa y en esta tarea participan directa o indirectamente la presencia de distintos actores, como la familia, los grupos de pares, las instituciones educativas, el rol protagónico que realizan las autoridades locales, regionales y los medios de masas (Escalante \& Alza, 2020).

En relación con lo afirmado, el hecho lamentable que desencadena el problema en cuestión, son las familias que utilizan la violencia como método y practica de crianza, grupos de pares con comportamiento violento. Además, de la educación básica y universitaria que no están orientadas a formar competencias ciudadanas para el mundo de hoy, también existe un desinterés por parte de los entes locales, regionales por establecer un vínculo con las instituciones educativas y las necesidades. 
Cuando se hace mención a las familias que utilizan la violencia como método y práctica de crianza, muchas familias han implementado un sistema de educación basado en la disciplina violenta que incluye agresión física (castigo corporal o físico) y psicológica vulnerando la convivencia pacífica de las familias (UNICEF, 2019). En el mundo hay aproximadamente 300 millones de niños entre dos y cuatro años, (3 de cada 4 menores) han sido sometidos a disciplina violenta por sus tutores y 250 millones han sido sometidos a castigos físicos; en otros casos, existen niños que viven en hogares donde la violencia doméstica es parte del día. Por ejemplo, 176 millones de niños menores ( 1 cada 4 menores) son víctimas constantes por parte de sus madres que también son violentadas (UNICEF, 2017).

En Perú, en relación a la violencia física (UNICEF, 2019) afirma que 6 de cada 10 niñas, niños y adolescentes fueron golpeados en algún momento en sus hogares, de los cuales $61 \%$ han experimentado jalones de pelo o de orejas, $21 \%$ abofetearon, mordieron, les castigaron con puñetes, con correa, cuerdas o palos y el $2 \%$ sufrió ataques con cuchillos u otras armas. En cuanto a la violencia psicológica, aproximadamente un $70 \%$ de menores de edad fueron víctimas de amenazas (17\%), humillaciones (33\%), desaprobación (30\%), insultos ( $42 \%$ ), apodos ofensivos (25\%) y burlas $(16 \%)$.

Según el INEI (2019) indica que durante el periodo de enero a mayo de 2019, los agresores de violencia familiar lo constituyeron hombres en un $83,1 \%$ y mujeres en $16,9 \%$. Además, Escalante \& Alza (2020) afirman que existen muchos grupos de pares con ideas cuestionadoras en oposición a los sistemas formales, estudiantes y jóvenes que anteceden sus necesidades, prioridades e intereses vulnerando los derechos de los demás. UNESCO (2019) reporta que, a nivel global, el 32\% de estudiantes fueron víctimas de alguna forma de acoso perpetrado por sus pares. Uno de cada tres niños (as) es víctima de acoso y violencia física, siendo el más frecuente el acoso físico, seguido del sexual. El $16 \%$ de estudiantes fueron golpeados, pateados, empujados y encerrados en espacios; el $11 \%$ fueron objeto de burlas, comentarios o gestos de índole sexual y el 5,5\% fueron ignorados o excluidos de actividades intencionadas. Comportamientos que afectan de sobremanera el bienestar, la dignidad personal y la buena convivencia.

Asimismo, en Perú, UNICEF (2019) señala que el SISEVE.PE reporta que un total 26,285 casos de violencia se han dado en la escuela, del cual el $54 \%$ ocurre entre escolares y $46 \%$ por los colaboradores de las empresas educativas. INEI (2019) agrega que de enero a mayo de 2019 las fuerzas del orden interno (PNP) registraron 3,236 acusaciones por violencia sexual, siendo las regiones de: Lima $(1,038)$, Arequipa (339), Cuzco (204), Junín (195), La Libertad (184) y Lambayeque (155), donde el 93,4\% de las denuncias por violencia sexual son contra la mujer, observándose una clara evidencia de falta de conciencia cívica, respeto por la dignidad y los derechos del otro.

La limitada formación de las competencias ciudadanas también es producto de los altos niveles de corrupción de las autoridades de turno. En América Latina, un tercio de los países presentan altos niveles de corrupción entre los que destacan: Cuba, Guatemala, Honduras, Venezuela, Nicaragua, Haití y República dominicana (IDEA, 2020). De igual forma, CADE Educación (2019) indica que el Perú está viviendo una profunda crisis institucional que limita el sentido de pertenencia, el respeto a los principios y valores democráticos, las autoridades no tienen la intención de asumir responsabilidades en pro del bien común.

Una educación básica y universitaria que no están orientadas a formar competencias ciudadanas tan requeridas en el mundo de hoy. América Latina y el Caribe cuenta con las más grandes brechas del mundo en cuestión de habilidades, debido al sistema de formación de talento guiados por currículos instructivos, desfasados, desconectados de la realidad (Mateo et al., 2019) excesivamente sobrecargados, fragmentados, escuelas aletargadas, que no responden rápidamente a los cambios, con una educación formal y docentes enfocados a una ciudadanía pasiva, solo al cumplimiento de deberes y derechos, a espalda del contexto local, regional, nacional e internacional (Escalante \& Alza, 2020).

Evidencia clara es lo que señalan Torres-Barreto et al. (2019), tomando en cuenta la información del periodo ICFES 2017, cuyos resultados reflejan que los estudiantes colombianos participan de manera 
mínima en política, civismo; además, de poseer docentes con limitada formación en competencias ciudadanas. Zambrano (2018) indica que los alumnos universitarios de la ciudad de Magdalena (Colombia) demostraron que las prácticas educativas están cargadas de exposiciones y que no contribuyen al impulso de las competencias cívicas, con esta actividad el estudiante está impedido de expresar sus puntos de vista, reflexionar sobre sus experiencias, manifestar sus emociones, mostrar asertividad, empatía, buena comunicación y acciones ciudadanas en el aula.

Según Álvarez et al. (2019) afirmaron que algunas universidades presentan vacíos y debilidades entre el currículo establecido (objetivos, perfiles y asignaturas) y los resultados en el conocimiento de las áreas que constituyen competencias ciudadanas. Así, lo confirman Álvarez et al. (2018) al sustentar en sus resultados que un $\mathbf{7 7 . 7 8 \%}$ de los alumnos de derecho de una universidad de Colombia coinciden en que aún no existe coherencia entre el desarrollo de competencias y la práctica de estas, además, de existir descuido para resolver conflictos, hay alianzas establecidas entre compañeros, no desean trabajar con diferentes estudiantes, en este sentido; urge la necesidad de insertar en las instituciones asignaturas afines que estén orientados a educar en la civilidad y sana convivencia.

Ante esta problemática, cabe preguntarse ¿Cuáles son las definiciones de competencias ciudadanas que proponen los artículos analizados?, ¿cuáles son los fundamentos epistemológicos en las que se sustentan las investigaciones sobre formación de competencias ciudadanas?, ¿por qué desarrollar competencias ciudadanas en el siglo XXI?, ¿cuáles son las competencias ciudadanas que proponen los investigadores para responder a un mundo globalizado?, ¿en qué países se han realizado estudios sobre competencias ciudadanas?,

En este sentido, el problema queda formulado: ¿Cuáles son las propuestas o intervenciones que se hacen sobre las competencias ciudadanas en los artículos revisados? Asimismo, el trabajo tiene como objetivo: realizar un análisis sistemático de los estudios sobre formación de competencias ciudadanas para un mundo global.

Las razones que motivan la investigación son de carácter social y epistemológico. El primero, es que el problema surge de una situación real y está vinculada con la formación limitada de las competencias ciudadanas de los estudiantes. Muchos países de la región se enfrentan a casos de violencia, conflictos e irrespeto a las leyes. La mayoría de los jóvenes no tienen clara la idea de patria, identidad y formación ciudadana. En segundo lugar, porque la investigación toma en cuenta los aportes de diferentes especialistas que, desde su experiencia y enfoque, analizan el objeto de estudio de acuerdo con ciertas metodologías que serán evaluadas para respuesta a las preguntas planteadas.

\section{Marco teórico \\ Competencias ciudadanas}

Cuando se recurre a definir las competencias ciudadanas, Torres-Barreto et al. (2019) señalan que es el conjunto de saberes cognitivos, actitudinales y de habilidades integrales, de naturaleza transversal, que posibilitan al ciudadano actuar de manera constructiva en sociedades democráticas y modernas. Para Paba-Medina et al. (2020) estas competencias también incluyen el desarrollo interconectado de habilidades cognitivas, emocionales y comunicativas, necesarias para desarrollarse desde una edad temprana hasta la universidad, como parte de la vida académica y social (León, 2020) y (Carreño y Rozo, 2020), permitiéndole actuar de manera constructiva, así como responsable (Álvarez et al., 2018).

Asimismo, se consideran capacidades efectivas de actuación en sociedades y estados democráticos (Alvarez et al., 2019). Precisamente su formación en estos tiempos se vuelve vital: Primero, porque contribuye al desarrollo de hábitos democráticos, al fortalecimiento de la ciudadanía, a la construcción de una cultura política, cívica y moderna; Segundo, por el enfoque pedagógico propuesto en la formación de competencias incluye el saber hacer, ser y convivir, donde se transluce en toda la conducta y comportamiento de civismo. Alvarez et al. (2017) argumenta que las competencias ciudadanas influyen 
en la formación de la ciudadanía; puesto que, la última instancia de su expresión se concreta en la acción; en esa misma línea, Carrillo et al. 2018) resalta la importancia de adquirir habilidades, conocimientos y actitudes cívicas para un mejor actuar. Cabrera-Jiménez (2020) señala que para consolidar el bien común, colectivo, global, así como el respeto a la normatividad y reconocimiento de derechos y deberes se debe de institucionalizar la democracia con agentes civiles.

\section{Fundamentos de la formación de competencias ciudadanas}

Los trabajos analizados se abordan desde el fundamento legal, social y empresarial: desde la perspectiva legal, se encontró la Ley Orgánica de Educación, LOE, 2006, en España que considera aspectos fundamentales en torno a la adquisición de capacidades y competencias.

En Chile está la ley General de Educación, LGE, 2009; Decreto 452, 2013, que establece que la formación profesional se construye articulando el dominio de las competencias de especialidad con la formación general de educación media y las competencias ciudadanas (Carrillo et al., 2018).

Desde el ámbito pedagógico, Parraguez (2021) propone lineamientos para acercar la escuela a la ciudadanía, Núñez et al. (2020), Zambrano (2018) y Álvarez et al. (2018) plantean que las competencias ciudadanas se pueden trabajar y desarrollar a partir de la aplicación de talleres, ensayos, trabajos de investigación y estrategias de aprendizaje. Alvarez et al. (2017) consideran que se realice con estrategias de formación sistémica del alumno consistente en la introducción de una catedra virtual y Avendaño et al. (2016) con un modelo que permita formar en la escuela ciudadanos por medio del trabajo en aula, principios, manejo de contenidos y el sistema de evaluación.

Desde la didáctica está lo propuesto por Paba-Medina et al. (2020) con el desarrollo de una herramienta didáctica, motivacional propia de la gamificación, elaborada para entornos virtuales y con la finalidad de optimizar el proceso docente educativo (PEA) de las competencias ciudadanas. El trabajo de Jaime et al. (2019) proponen el uso de la radio como un medio. Fuentes-Moreno et al. (2020) recomiendan trabajarlo con la Historia, Maldonado (2018), Calle-Alvarez y Lozano-Prada (2019) desde el aula y articulando el uso de las TIC al proceso de aprendizaje con el desarrollo de competencias para la educación superior y básica. Por último, Carreño y Rozo (2020), que proponen estrategias de prevención, participación, intervención y estrategias basadas en las TIC.

\section{Educar en competencias ciudadanas}

Cabrera-Jiménez (2020) y Alvarez et al. (2019) señalan que la necesidad de trabajar la formación ciudadana y fortalecer el comportamiento cívico se ha vuelto recurrente e importante en el contexto internacional como regional desde los noventa hasta el presente siglo, precisamente por las siguientes razones: el contexto y la formación de competencias, pues el mundo actual requiere una mayor formación y preparación de saberes integrales con el desarrollo de competencias que ayuden a resistir el cambio y lograr la adaptabilidad (Maldonado, 2018).

En esa línea, los investigadores recomiendan trabajar competencias que permitan responder para la vida (León, 2020), que ayuden a insertarse al mundo laboral, competencias integrales, transversales, básicas para las relaciones sociales, cívicas y ciudadanas (Carrillo et al., 2018), que ayuden a responder a los cambios coyunturales (Zambrano, 2018) y educar pensando en el desarrollo de capacidades para la vida en correspondencia con la cultura, tradición, demandas sociales (Morales et al., 2021) y la promoción del desarrollo sostenible (Paba-Medina et al., 2020).

\section{Demanda de cultura de paz y convivencia ciudadana}

La sociedad exige la formación de ciudadanos en una cultura de paz (ONU, 2018) y (CADE Educación, 2019). A medida que pasa la sociedad global atraviesa momentos críticos, con violencia, contaminación ambiental, corrupción institucional, tensiones globales, cambios sociales, guerras, crisis de salud, desigualdades, incertidumbres vulneración de derechos, escaso cumplimiento de los deberes. 
En ese sentido, Carreño y Rozo (2020) afirman que se hace necesario contar con jóvenes con competencias ciudadanas que desarrollen el compromiso no solo con su sociedad, sobre todo consigo mismos. Con la participación de ciudadanos activos, solidarios, responsables que demuestren una ciudadana sustantiva (Manuel, 2017), personas que sepan ejercer una ciudadanía activa, crítica y prospectiva, capaces de analizar la realidad social y gestionar sus sentimientos (Núñez et al., 2020).

El presente siglo, está centrado en una educación de calidad con aprendizajes que involucre la formación ciudadana; la mejora de la convivencia basada en el respeto a la diversidad cultural, los valores familiares y los derechos humanos (García, 2018). Por tanto, el gran reto de las instituciones educativas es desarrollar en los estudiantes competencias ciudadanas que hagan de las personas sujetos solidarios, empáticos, capaces de respetar sus propios derechos humanos y los de los demás, con el objetivo de construir una sociedad participativa y democrática e inclusiva, capaz de trabajar en equipo para alcanzar metas y objetivos comunes (Calle-Alvarez y Lozano-Prada, 2019) y esto se logrará alineando la gestión de los programas educativos con la formación ciudadana (Parraguez, 2021), y en las universidades también se propone trabajar la competencia cívica con la creación de escenarios intactos con el fin de convivir, respetar la diversidad y la dignidad humana (Avendaño et al., 2016).

\section{Competencias ciudadanas requeridas por el mundo globalizado}

Las competencias ciudadanas para el mundo global resaltadas en los artículos revisados son: comunicación, intervención en conflictos, trabajo en equipo (Alvarez et al., 2017) participación e interdisciplinariedad (Carreño y Rozo, 2020), convivencia y paz, participación, resolución de conflictos y pluralidad (Monterrosa, 2020). Además, Núñez et al. (2020) agregan otras tales como: el juicio moral, la empatía, la argumentación, el diálogo, la coordinación de perspectivas, la descentralización y el pensamiento sistémico; Jaime et al. (2019), señalan a la convivencia, la paz, la participación, responsabilidad democrática, pluralidad e identidad como las más requeridas y para Zambrano (2018) la empatía y las habilidades cognitivas.

Por otro lado, Paba-Medina et al. (2020) afirman que las habilidades requeridas son: cognitivas (multiperspectivismo, argumentación, y pensamiento sistémico), las comunicativas (expresión oral y escrito), las emocionales (manejo de la empatía y las emociones) e integradoras y Mescua et al. (2020) para esta última presentación, añade las competencias de conocimientos que son las que implican el dominio sobre el conocimiento de los derechos esenciales.

\section{Metodología}

El estudio se ubica en los principios metodológicos de los artículos de revisión sistemática y metaanálisis, en cuanto a ello Merino-Trujillo (2013), Ferreira et al. (2011) y Vera (2017), lo definen como un artículo especializado, detallado, selectivo y crítico, orientado a examinar y sintetizar las publicaciones disponibles en un tema específico con la pretensión de responder a una interrogante y situarla en alguna perspectiva. Metodológicamente, se enmarcó dentro de la investigación teórica, que según CONCYTEC (2018) es una tipología cuya finalidad no es medir, sino generar un conocimiento completo del problema o tema específico, a través de la interpretación de aspectos fundamentales o de las relaciones que se establecen.

La planificación metodológica se desarrolló siguiendo el procedimiento general del metaanálisis que consistió en: definir la variable, elegir los términos de búsqueda, revisar sistemáticamente la literatura, analizar los datos, evaluar la heterogeneidad de los documentos e interpretar los resultados (Bolaños y Calderón, 2014). 


\section{Figura 1}

Procedimiento metodológico de la revisión sistemática y meta-análisis.

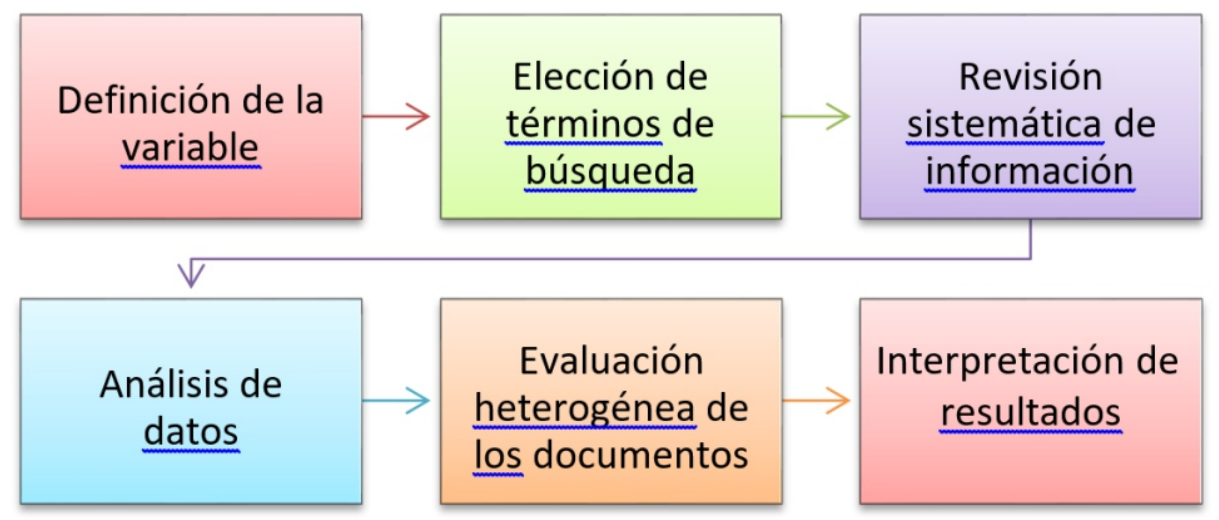

La definición de la variable "formación de competencias ciudadanas", se estableció en relación con la necesidad objeto de estudio encontrado en el proceso formativo y corresponde a una variable de naturaleza cualitativa. La elección de los términos de búsqueda, se realizó con el apoyo de dos tesauros. En primer lugar, se utilizó la "terminología controlada y estructurada" del Tesauro multidisciplinario de la UNESCO considerando tres opciones (tipo, grupo y parentesco). En segundo lugar, se aprovechó los descriptores jerárquicos del Tesauro Europeo de la Educación a través del aprendizaje asociativo y ciencias sociales.

La revisión sistemática, consistió en la búsqueda exhaustiva de artículos científicos originales en base a las preguntas cientíicas, proceso que se realizó con el objetivo de identificar los artículos potenciales en los últimos cinco años a nivel global, latinoamericano y nacional, además, la estrategia se llevó a cabo tomando la variable de estudio y para una búsqueda más efectiva se utilizó descriptores de búsqueda y palabras clave tales como: competencias para la vida, competencias ciudadanas, ciudadanía global y educación global en proveedores de información y diversas bases de datos cientíicos indizadas en: ProQuest, Ebsco, SciELO, Scopus, Concytec, Dialnet.

Asimismo, con la ayuda de términos y operadores booleanos, se realizó una meta búsqueda en el Google Scholar, encontrando así informes que describen los problemas de ciudadanía global publicados por organismos e instituciones nacionales e internacionales como UNESCO, CEPAL, UNICEF, INEI, International IDEA y CADE-IPAE también de losúltimosaños.

Para el procedimiento del análisis de los datos, se organizó una síntesis cualitativa de los datos y metadatos en una tabla de valores de refinamiento: autor, título, nombre de la revista, fecha y lugar de publicación, palabras clave, objetivo, identificador de artículos digitales DOI, metodología utilizada y hallazgos encontrados; parámetros que permitieron realizar el cotejo, decidir la elegibilidad de las publicaciones, evitar la duplicidad y el análisis respectivo. Por razones de factibilidad, la evaluación heterogénea consistió en incluir artículos indexados relevantes para el meta-análisis, proceso que se llevó considerando criterios de selección: inclusión y exclusión a los títulos y resúmenes obtenidos. Según los criterios de inclusión, se optaron por elegir 34 de los 54 estudios analizados desde el año 2016 al 2021, de los cuales, 23 artículos fueron en español, 2 en inglés, indexados en revistas con alto factor de impacto y 09 informes de instituciones internacionales y nacionales. También se consideró publicaciones con enfoque cualitativo, cuantitativo y mixto, los mismos que suscitaron a la reflexión, sistematización y análisis. Con respecto a los límites de la revisión sistemática (criterio de exclusión) no se consideraron publicaciones con falencias de redacción y estructura, aquellos de dudosa procedencia o publicados en bases de datos de acceso libre.

Considerando la intencionalidad del meta-análisis, la interpretación de los resultados se realizó en función a la evaluación heterogénea de cada unidad de análisis establecida en la fase de selección, proceso que se llevó a cabo con la ayuda estructurada y explicita de diversas tablas construidas con diversos criterios. Primero, identificación de artículos originales y revistas indizadas en base de datos. Segundo, publicaciones en base a la variable, objetivo y tipo de investigación. Tercero, publicaciones según campo de acción y tipo de investigación. Cuarto, artículos publicados según país y año. 
De acuerdo al razonamiento de abducción e inducción de Peirce, la construcción de tablas permitió la adopción provisional de una inferencia explicativa, sometiéndose a una contrastación de tal forma que permita ver las concordancias, discordancias y complementariedad de los estudios en cuestión. Los resultados obtenidos se presentan a través de tablas y figuras expresadas como resúmenes de todos los resultados, que surgió de cada estudio seleccionado que se empleó como insumo para cotejar las posturas y resultados obtenidos.

Los artículos seleccionados para meta-análisis conforman un promedio de 25 revistas científicas indizadas en base de datos dealto impacto, entre ellas: Revista Academia y Virtualidad Latindex Educativa, Rev. Ciencias Sociales Latindex. Redalyc Org, Educación y Educadores, Revista Eleuthera, Panorama, Revista Eduser, Podium Revista de Ciencia y Tecnología en la Cultura Física, Edumecentro, Espacios, Revista Chilena de Ortopedia y Traumatología, Revista De Ciencias Sociales-Costa Rica, Telos Revista Enfoques Educacionales, Salud En Tabasco, Revista Electrónica-Investigación Educativa, Revista de Estudios Interdisciplinarios En Ciencias Sociales, Sophia, Agora U.S.B, Information and Modeling, Revista de Pedagogía, Revista Española de Cardiología, Revista Boletín Redipe, Revista Educación Educadores de La Universidad de Sabana, Revista Española deOrientación y Psicopedagogía, Sciences Communications.

En cuanto a las limitaciones presentadas, es que las publicaciones realizadas a nivel peruano son escasas, también se encontró artículos con información incompleta, trabajos que no respondían a las preguntas planteadas y objetivos formulados, artículos en otros idiomas. Sin embargo, a pesar de esto, la realización de este artículo resulta importante ya que, al ser un trabajo de revisión sistemática, presenta el análisis realizado de distintas investigaciones de informes y artículos originales de la variable competencias ciudadanas en estudiantes, convirtiéndose en una valiosa fuente de información para las investigaciones futuras.

\section{Resultados}

\section{Tabla 1}

Identificación de publicaciones científicas

\begin{tabular}{ccc}
\hline Publicaciones & Frecuencia & $\%$ \\
\hline Artículos & 25 & 74 \\
Informes & 9 & 26 \\
Total & 34 & 100 \\
\hline
\end{tabular}

Fuente: tabla de valores de refinamiento.

\section{Tabla 2}

Artículos encontrados en base de datos y proveedores de información

\begin{tabular}{ccr}
\hline $\begin{array}{c}\text { Base de datos Proveedor } \\
\text { de información }\end{array}$ & Número de artículos & $\%$ \\
\hline PROQUEST & 10 & 18.5 \\
EBSCO & 10 & 18.5 \\
SCIELO & 8 & 14.8 \\
SCOPUS & 6 & 11 \\
CONCYTEC & 7 & 12.9 \\
GOOGLE ACADÉMICO & 8 & 14.8 \\
DIALNET & 5 & 9 \\
TOTAL & 54 & 100 \\
\hline
\end{tabular}

Fuente: tabla de valores de refinamiento. 


\section{Tabla 3}

Informes en repositorios de organismos internacionales y nacionales seleccionados

\begin{tabular}{cccc}
\hline Organismos & $\begin{array}{c}\text { Año de } \\
\text { publicación }\end{array}$ & Informes & $\%$ \\
\hline UNESCO & 2021 & 1 & 11.1 \\
CEPAL & 2020 & 1 & 11.1 \\
International IDEA & 2020 & 1 & 11.1 \\
UNICEP & $2017 / 2019$ & 2 & 22,2 \\
BID & 2019 & 1 & 11,1 \\
ONU & 2018 & 1 & 11.1 \\
IPAE & 2019 & 1 & 11.1 \\
INEI & 2019 & 1 & 11.1 \\
TOTAL & & 9 & 100 \\
\hline
\end{tabular}

Fuente: tabla de valores de refinamiento.

\section{Tabla 4}

Publicaciones de artículos seleccionados por país y año

\begin{tabular}{ccccc}
\hline Países & $\begin{array}{c}\text { Total de } \\
\text { publicaciones }\end{array}$ & $\%$ & $\begin{array}{c}\text { Año de } \\
\text { publicación }\end{array}$ & $\begin{array}{c}\text { Número de } \\
\text { Publicaciones }\end{array}$ \\
\hline & & & 2016 & 1 \\
COLOMBIA & 16 & 64 & 2017 & 3 \\
& & & 2018 & 5 \\
& & & 2019 & 2 \\
MEXICO & 2 & 8 & 2017 & 5 \\
& 2 & & 2018 & 1 \\
ESPAÑA & & & 2018 & 1 \\
& 2 & 8 & 2021 & 1 \\
PERÚ & 1 & 4 & 2020 & 2 \\
CUBA & 1 & 4 & 2021 & 1 \\
CHILE & 1 & 4 & 2021 & 1 \\
BOLIVIA & 25 & 100 & TOTAL & 25 \\
TOTAL & & & & 1 \\
\hline
\end{tabular}

Fuente: tabla de valores de refinamiento.

\section{Tabla 5}

Publicaciones en base al campo de acción y tipo de investigación

\begin{tabular}{clrr}
\hline \multicolumn{1}{c}{ Criterio } & \multicolumn{1}{c}{ Publicaciones } & $\mathrm{F}$ & $\%$ \\
\hline \multirow{2}{*}{ Campo de acción } & $\begin{array}{l}\text { Competencias ciudadanas en estudiantes } \\
\text { universitarios }\end{array}$ & 17 & 68 \\
& $\begin{array}{l}\text { Competencias ciudadanas en educación } \\
\text { básica } \\
\text { Total }\end{array}$ & 8 & 32 \\
& Cuantitativas & 25 & 100 \\
Tipo de investigación & Cualitativas & 11 & 44 \\
según su enfoque & Mixtas & 9 & 36 \\
& Total & 5 & 20 \\
\hline
\end{tabular}

Fuente: tabla de valores de refinamiento. 


\section{Discusión}

Existe una marcada coincidencia en la conceptualización de las competencias ciudadanas en los trabajos de Paba-Medina et al. (2020), León (2020), Núñez et al. (2020), Carreño y Rozo (2020), Álvarez et al. (2018) y Zambrano (2018) quienes lo conciben como una combinación articulada de saberes cognitivas, operaciones emocionales y comunicativas para promover la formación integral de los alumnos.

Desde el campo de la didáctica Paba-Medina et al. (2020) afirman que una de las formas de estimular la motivación para el aprendizaje y el perfeccionamiento de las competencias ciudadanas es recurrir a la gamificación, esto es complementado por Maldonado (2018) al precisar que los procesos pedagógicos deben apuntar a potenciar capacidades, habilidades y destrezas de los estudiantes. Para ello, las instituciones educativas deben utilizar métodos flexibles como las que proporciona las TIC, una herramienta tecnológica que contribuye al refuerzo de la criticidad, la interpretación, comunicación y transformación.

En cuanto, a las competencias ciudadanas a desarrollar, fue posible encontrar ciertas coincidencias entre lo planteado por Carreño y Rozo (2020) y Monterrosa (2020) sobre la propuesta de desarrollar la convivencia, pero este último autor agrega la pluralidad como un eje sobre el que debemos trabajar para fomentar la tolerancia, el respeto a las ideas, creencias, costumbres y tradiciones.

Respecto a las estrategias que ayudarían a potenciar las competencias ciudadanas están los talleres de juegos de negociación (TJN) que incluyen juegos de inversión, de confianza, normas sociales, negociación de conflictos, foros virtuales en una red social, el debate competitivo (Núñez, Zambrano, et al., 2020), complementando lo afirmado también está Paba-Medina et al. (2020), León (2020), y la estrategia de negociación, mesas de diálogo, proyectos de aula, la elaboración de manuales de convivencia, el uso de la TIC y sus aplicaciones (Carreño y Rozo, 2020), talleres, artículos, estudios de casos, esquemas, resúmenes, trabajos de investigación, síntesis (Álvarez et al., 2018), los círculos de paz, con el apoyo de un profesional que oriente el conflicto hacia la resolución pacífica del problema, la creación de una catedra especial sobre competencias ciudadanas, conversatorios que promuevan habilidades cívicas, proyectos de aula, la bitácora de experiencias del conflicto y posconflicto (Álvarez y Moreno, 2017), que serían nuevas opciones de trabajo para buscar la promoción y fortalecimiento de competencias cívicas.

Otro punto de coincidencia está en la implementación de una catedra desarrollada a lo largo de varios semestres (Álvarez et al., 2018) y que según Álvarez y Moreno (2017) debería ser una asignatura específica de competencias cívicas denominada ciudadanía responsable, convirtiéndose en una estrategia esencial que ayuda a promover las competencias ciudadanas entre los estudiantes universitarios.

Asimismo, Núñez et al. (2020) y Zambrano (2018) coinciden en reconocer la importancia que tienen las estrategias o metodologías que utiliza el maestro en el aula, pues gracias a ellas, se logrará mayor participación de un estudiante ciudadano universitario, pero también es esencial que estas estrategias sean constantemente evaluadas, revisadas, innovadas y renovadas, sin embargo, en cuanto a las propuestas realizadas por Núñez et al. (2020), se enfocan únicamente en el juego de negoción (TJN) como estrategia pedagógica que incluye 4 juegos como es la inversión, Garrote y zanahoria, conflicto, mientras que Zambrano (2018) propone prácticas, constructivas (estudio de casos), de profundización y lúdicas. Por otro lado, PabaMedina et al. (2020) se inclina en plantear una herramienta para entornos virtuales utilizando la gamificación.

\section{Conclusiones}

Las competencias ciudadanas son una combinación de saberes integrales (funciones cognitivas, habilidades y actitudes), pueden desarrollarse a lo largo de la vida y permiten una convivencia sana y democrática.

Formar competencias ciudadanas en las instituciones educativas es una tarea fundamental, ya que les permitirá afrontar las nuevas demandas del mundo global (ciudadanos competentes, trabajadores en equipo, personas sensibles, seres empáticos, individuos responsables, con una cultura de paz y convivencia ciudadana). 
Frente a una sociedad mundial que atraviesa momentos críticos, con violencia, contaminación ambiental, corrupción institucional, tensiones globales, cambios sociales, guerras, crisis de salud, desigualdades, incertidumbres, vulneración de derechos y escaso cumplimiento de deberes, es de vital importancia trabajar en investigaciones que promuevan la convivencia sana y el bien común.

A nivel global, las organizaciones internacionales han publicado informes con la finalidad de desarrollar una cultura de paz. Ante esto, los artículos que destacan son cuantitativos, cualitativos y mixtos, la mayoría de los cuales apuntan a fortalecer las competencias ciudadanas en diferentes niveles: básico y superior, con énfasis en Colombia.

La mayoría de estudios insisten y enfatizan el desarrollo de programas, especialmente cursos curriculares, tanto en educación básica como en educación superior con el fin de formar las competencias ciudadanas.

\section{Referencias}

Alvarez, A. A.; Pertuz, O. de J.; Sandoval, J.; Saker, G. C. \& Gómez, M. (2017). Competencias Ciudadanas para la formación integral en educación superior. https://www.researchgate.net/publication/320391163\%0ALIBRO-

Álvarez, A. A.; Sandoval, O. J. \& Puello, M. (2018). Estrategias pedagógicas para desarrollar competencias ciudadanas en estudiantes de Derecho. Espacios, 39(29), 6-14. https://repositorio.cuc.edu.co/bitstream/ handle/11323/1613/Estrategias pedagógicas para desarrollar competencias ciudadanas en estudiantes de Derecho.pdf? sequence $=1$

Alvarez, A.; Valencia, A. \& López, H. (2019). Competencias ciudadanas en sedes regionales de la universidad del valle: avances y dificultades en el proyecto de formación ciudadana. Boletín REDIPE, 8, 44-71. https://doi.org/https://doi.org/10.36260/rbr.v8i4.727

Avendaño, W. R.; Paz Montes, L. S. \& Parada Trujillo, A. E. (2016). Construcción de ciudadanía: un modelo para su desarrollo en la escuela. Agora U.S.B., 16(2), 479. https://doi.org/10.21500/16578031.2444

Bolaños, R. \& Calderón, M. (2014). Introducción al meta-análisis tradicional. Revista de Gastroenterología Del Perú, 34(1), 45-51. http://www.scielo.org.pe/scielo.php?script=sci_arttext\&pid=S1022-51292014000100007\&lng $=$ es\&nrm=iso\&tlng=es

Cabrera-Jiménez, M. F. (2020). Relevancia de las competencias ciudadanas en construcción de civilidad en educación superior. Revista Educación y Humanismo, 22(38), 1-24. https://doi.org/http://dx.doi.org/10.17081/eduhum.22.38.3555

CADE Educación. (2019). Encuesta de Habilidades al Trabajo (ENHAT) 2017-2018: Causas y Consecuencias de la Brecha de Habilidades en Perú. Banco Interamericano de Desarrollo (IPAE (ed.)). https://doi.org/http://dx.doi.org/10.18235/ 0001653

Calle-Alvarez, G. Y. \& Lozano-Prada, A. (2019). La alfabetización digital en la formación de competencias ciudadanas en la básica primaria. Eleuthera, 20(2018), 35-54. https://doi.org/10.17151/eleu.2019.20.3

Carreño, M. \& Rozo, H. (2020). Estrategias para desarrollar la convivencia y la paz desde la educación. Academia y Virtualidad, 13(2), 35-56. https://doi.org/10.18359/ravi.4501

Carrillo, O.; Jurado, P. \& Lagos, P. (2018). Las competencias ciudadanas en la formación profesional de las ciudades de Concepción (Chile) y Barcelona (España)/Citizen competences in the profesional training in the cities of Concepción (Chile) and Barcelona (España). REOP - Revista Española de Orientación y Psicopedagogía, 29(3), 110.https://doi.org/10.5944/reop.vol.29.num.3.2018.23324

CEPAL-UNICEF. (2020). Violencia contra niñas, niños y adolescentes en tiempos de COVID-19. https://repositorio.cepal.org/bitstream/handle/11362/46485/1/S2000611_es.pdf

Escalante, H. \& Alza, V. (2020). Citizen participation and improvement of basic education in the districts of Laredo, Simbal and Poroto. Trujillo Province. Revista Ciencia y Tecnología, 16(3), 131-142. https://doi.org/10.17268/rev.cyt.2020.03.13

Ferreira, I.; Urrútia, G. \& Alonso-Coello, P. (2011). Systematic reviews and meta-analysis: Scientific rationale and interpretation. Revista Española de Cardiología, 64(8), 688-696. https://doi.org/10.1016/j.recesp.2011.03.029

Fuentes-Moreno, C.; Sabariego-Puig, M. \& Ambros-Pallarés, A. (2020). Developing social and civic competence in secondary education through the implementation and evaluation of teaching units and educational environments. Humanities and Social Sciences Communications, 7(1). https://doi.org/10.1057/s41599-020-0530-4 
García, J. (2018). Diversas perspectivas de formación ciudadana en bachillerato y una propuesta de la cultura ciudadana. Revista De La Escuela De Ciencias De La Educación, 2(13), 56-77. https://doi.org/https://doi.org/10.35305/rece.v2i13.384

Huanca-Arohuanca, J.; Supo-Condori, F.; Sucari, R. \& Supo Quispe, L. (2020). El problema social de la educación virtual universitaria en tiempos de pandemia, Perú. Innovaciones Educativas, 22(Especial), 115-128. https://doi.org/10.22458/ie.v22iespecial.3218

IDEA. (2020). Balance de las tendencias democráticas en América Latina y el Caribe antes y durante la pandemia de la COVID-19. In Balance de las tendencias democráticas en América Latina y el Caribe antes y durante la pandemia de la COVID-19. https://doi.org/10.31752/idea.2020.69

INEI. (2019). Indicadores de Violencia familiar y sexual 2012-2019. Journal of Chemical Information and Modeling, 2(9), 1-81. https://www.inei.gob.pe/media/MenuRecursivo/publicaciones_digitales/Est/Lib1686/

Jaime, M. F.; Caicedo, M. C. \& Trujillo, I. C. (2019). A Radio Program: a Strategy to Develop Students' Speaking and Citizenship Skills. How, 26(1), 8-33. https://doi.org/10.19183/how.26.1.510470

León, F. (2020). Competencias ciudadanas: análisis crítico y pautas para la formación profesional. Asociación Latinoamericana de Sociología, 12(21), 369-388. https://uy.vlex.com/vid/competencias-ciudadanas-analisiscritico-857513067

Ley que modifica diversos artículos de la Ley 28303, Ley Marco de Ciencia, Tecnología e Innovación Tecnológica; y de la Ley 28613, El Peruano 4 (2018). https://busquedas.elperuano.pe/normaslegales/ley-que-modificadiversos-articulos-de-laley-28303-ley-mar-ley-n-30806-1666491-1/

Maldonado, M. E. (2018). El aula, espacio propicio para el fortalecimiento de competencias ciudadanas y tecnológicas. Sophia, 14 número1, 41-50. https://doi.org/DOI: http://dx.doi.org/10.18634/ sophiaj.14v.1i.822

Manuel, J. I. (2017). Formative Processes of Citizen Expressions on a Mexican University. Revista De Ciencias Sociales-Costa Rica, 2017(158), 85-97. https://www.redalyc.org/articulo.oa?id=15354921006

Mateo, M; Buenadicha, C. \& Bustelo, M. (2019). Transversales en américa latina y el caribe el futuro ya está aquí ¿Por qué el individuo del siglo 21 es diferente? In BID. https://publications.iadb.org/es/habilidades-del-siglo-21desarrollo-de-habilidadestransversales-en-america-latina-y-el-caribe

Merino-Trujillo, A. (2013). Como escribir documentos científicos. Artículo de revisión. Salud En Tabasco, 19(3), 90-94. http://www.redalyc.org/articulo.oa?id $=48730715004$

Mescua, A. C.; Ramos, R. P.; Ramos, P.; Ramírez, Y. P.; Cruz Llerena, B. S. \& Caycho, F. A. (2020). Niveles de competencias ciudadanas en estudiantes universitarios de Ciencias de la Salud. Eduser, 7(1), 69-81. https://doi.org/10.18050/eduser.v7i1.2516

Monterrosa, N. M. (2020). Competencias ciudadanas: Ejes presentes en el sector estudiantil Colombiano. Telos Revista de Estudios Interdisciplinarios En Ciencias Sociales, 22(2), 376-386. https://doi.org/10.36390/telos222.09

Morales, L.; Salvador, R. L.; Mesa, N. \& Veitía, I. J. (2021). El desarrollo de habilidades para la vida desde la promoción de salud. Edumecentro, 13(1), 236-251.

Núñez, S. N.; Zambrano, A. M.; Palacio, L. A. \& Maldonado, J. F. (2020). Juegos de negociación: estrategia para formación de competencias ciudadanas en universitarios. Educación y Educadores, 23(2), 291-308. https://doi.org/10.5294/edu.2020.23.2.7

Núñez, S. N.; Zambrano, A. M.; Palacio, L. A. \& Maldonado, J. F. (2020). Juegos de negociación: estrategia para formación de competencias ciudadanas en universitarios. Educación y Educadores, 23(2), 291-308. https://doi.org/10.5294/edu.2020.23.2.7

ONU. (2018). Día Internacional de la Convivencia en Paz. Boletín de La Sociedad Geológica Mexicana, 4(1), 15-17. https://doi.org/10.18268/bsgm1908v4n1x1

Paba-Medina, M. C.; Acosta-Medina, J. K. \& Torres-Barreto, M. L. (2020). Prioritisation of Citizen Competencies in a Gamma Context. Panorama, 14(27), 51-70.

Parraguez, U. A. (2021). ¿Qué ciudadanía queremos? Lineamientos para la Formación Ciudadana en la escuela. Revista Enfoques Educacionales, 18(1), 132. https://doi.org/10.5354/2735-7279.2021.60914

Sandoval, O. de J. \& Moreno, G. C. S. (2017). Contexto y realidad emergente de las competencias hacia la formación integral: educación superior. In Competencias Ciudadanas para la Formación Integral en educacion superior ( $p$. 214). file:///C:/Users/manu_/Downloads/Competencias_ciudadanas_para_la_formacio (1).pdf 
Torres-Barreto, M. L.; Alvarez-Melgarejo, M. \& Acosta-Medina, J. K. (2019). Desarrollo de competencias ciudadanas en Colombia y en el mundo: contexto actual y estrategias empleadas Development of citizen competences in Colombia and in the world : current context and strategies used. Universidad Industrial de Santander, October. https://doi.org/10.13140/RG.2.2.18390.80962

UNESCO (2019). Behind the Numbers: Ending School Violence and Bullying. In Sustainable Development Goals: Education 2030. https://unesdoc.unesco.org/ark:/48223/pf0000366483

UNICEF (2017). Una situación habitual. https://www.unicef.org/dominicanrepublic/media/1636/file/Publicación \%7C Una Situación Habitual.pdf

UNICEF (2019). Cifras de la violencia hacia los niños, niñas y adolescentes en el Perú. Encuesta Nacional de Relaciones Sociales Del Perú - ENARES, 8. https://www.unicef.org/peru/sites/unicef.org.peru/files/201909/cifras-violencia-ninasninos-adolescentes-peru-2019.pdf

Vera, O. (2017). Artículos de Revisión. Revista Chilena de Ortopedia y Traumatología, 58(02), 033-033. https://doi.org/10.1055/s-0037-1606585

Zambrano, E. L. (2018). Prácticas pedagógicas para el desarrollo de competencias ciudadanas. Revista Electronica de Investigacion Educativa, 20(1), 69-82. https://doi.org/10.24320/redie.2018.20.1.1409

\section{Cómo citar este trabajo}

Sánchez Tarrillo, S. J., \& Aguinaga Vásquez, S. J. (2021). Formación de competencias ciudadanas para responder a un mundo global. EDUCARE ET COMUNICARE: Revista De investigación De La Facultad De Humanidades, 9(2), 3042. https://doi.org/10.35383/educare.v9i2.673

\section{Financiación}

El presente artículo no cuenta con financiación específica para su desarrollo y/o publicación.

\section{Conflicto de interés}

Los autores del artículo declaran no tener ningún conflicto de intereses en su realización.

(C) Los autores. Este artículo es publicado por la Revista Educare et Comunicare de la Facultad de Humanidades, Universidad Católica Santo Toribio de Mogrovejo.

Este es un artículo de acceso abierto, distribuido bajo los términos de la Licencia Creative Commons Atribución-NoComercial-CompartirIgual 4.0 Internacional (CC BY- NC-SA 4.0), que permite el uso no comercial, distribución y reproducción en cualquier medio, siempre que la obra original sea debidamente citada. 